\title{
Critical Limb Ischemia in Patients with End-Stage Renal Disease: Do Long-Term Results Justify An Aggressive Surgical Approach?
}

\author{
PETER S. DOVGAN, M.D., ALEXANDER D. SHEPARD, M.D., \\ and TIMOTHY J. NYPAVER, M.D.
}

\begin{abstract}
End-stage renal disease (ESRD) patients probably represent the most difficult group of patients vascular surgeons are called upon to treat for critical limb ischemia. Advanced lower extremity arterial occlusive disease and frequent comorbidities make infrainguinal arterial bypass grafting (IABG) in this population both technically and medically challenging. Results of IABG are far inferior to those in non-ESRD patients. The greatest limitation to limb salvage appears to be progressive tissue necrosis and infection despite a patent bypass graft, a problem nearly unique to ESRD. Clinical predictors of outcome for IABG in this population have not been well established. The site and extent of tissue loss, the presence of associated infection, and the degree of pedal level occlusive disease have been identified as potentially important predictors. Improving outcome for ESRD patients with critical limb ischemia requires the establishment of firm guidelines for IABG to avoid futile and risky attempts at bypass in individuals better served by primary amputation.
\end{abstract}

Keywords End-stage renal disease, arterial, infrainguinal, bypass, renal disease

Current techniques of infrainguinal arterial bypass grafting (IABG) have allowed vascular surgeons to maintain limb viability and restore function in a large percentage of patients presenting with critical limb ischemia. Coupled with forefoot amputations and innovative procedures for tissue coverage, IABG can save many limbs previously deemed unsalvageable. Nevertheless, there exist certain patients for whom the benefits of arterial reconstruction are less obvious and for whom primary amputation may provide a more logical approach. Patients with end-stage renal disease (ESRD) represent one of the most frequently encountered subsets of patients with critical limb ischemia in whom the decision to bypass or amputate can be especially challenging, even for the most experienced vascular surgeon.

P.S.D., Clinical Vascular Fellow; A.D.S., Senior Staff Surgeon, Clinical Associate Professor of Surgery, Lniversity of Michigan Medical School; T.J.N., Senior Staff Surgeon, Division of Vascular Surgery, Department of Surgery, Henry Ford Hospital, Detroit, MI.

Copyright (C) 1999 by Thieme Medical Publishers, Inc., 333 Seventh Avenue, New York, NT 10001, LSA.

Tel.: +1(212) 760-0888, Ext. 132.0894-8046/1999/E1521-5768(1999,12:01:0081-0092:PVS000047. 
ESRD is defined as a state of native renal function that necessitates renal replacement therapy (hemodialysis, peritoneal dialysis, or renal transplantation) for survival. Patients with ESRD currently comprise only a small proportion of patients undergoing lower extremity arterial reconstructions ( $6 \%$ in our institution), but this may change dramatically in the future. In 1998, approximately 300,000 patients were receiving renal replacement therapy in the United States ${ }^{1}$; that number is growing at an annual rate 6 to $7 \%{ }^{2}$ and will reach an estimated 600,000 patients by $2010 .^{3}$ The greatest increase in treatment with long-term dialysis has been among the elderly and those with diabetes, two groups at high risk for peripheral arterial occlusive disease (PAOD).$^{4,5}$ In addition, over the last 2 decades, the life expectancy of dialysis patients has slowly improved, resulting in an older population with potentially more advanced atherosclerotic disease. ${ }^{4}$ These data suggest an increasing burden of PAOD in patients with ESRD and a concomitant rise in future demand for arterial reconstruction.

Since the late 1980s, ESRD patients with critical limb ischemia undergoing IABG have been recognized as a subgroup with inferior graft patency and limb salvage compared to others treated with infrainguinal bypass. ${ }^{\circ}$ In addition, the comorbidities frequently associated with ESRD (e.g., diabetes, hypertension, and coronary artery disease) have an adverse effect on operative outcome as well as long-term survival. ${ }^{7}$ Amputation, however, may not represent a "safe" alternative for these patients as the mortality for lower extremity amputation in this population is substantial. ${ }^{8}$ The question vascular surgeons must consider is whether the results of LABG in this group justify the risks to the patient and the expense to the health care system.

\section{PERIPHERAL ARTERIAL OCCLUSIVE DISEASE}

Although good data are lacking on the incidence of PAOD in ESRD, extrapolation from coronary artery disease data would suggest a prevalence far in excess of that in patients without renal failure. Almost half of all deaths in ESRD patients are caused by cardiovascular disease, with cardiac arrest, acute myocardial infarction, and arrhythmia as the leading causes. ${ }^{9}$ The aggressive atherosclerosis present in many of these patients is the result of a number of well-established risk factors frequently associated with chronic renal failure: diabetes mellitus, hypertension, hyperlipidemia, hyperhomocystinemia, and advancing age. Diabetes mellitus or primary hypertension together account for almost two thirds of cases of ESRD. ${ }^{3}$ In addition, secondary hypertension occurs with many renal disorders and is nearly universal by the time dialysis is initiated. Lipid disturbances are common with chronic renal failure (uremic dyslipidemia) and are characterized by elevated levels of total cholesterol, lipoprotein (a), and triglycerides and reduced levels of high-density lipoprotein. This atherogenic lipid profile is further heightened by enhanced oxidation 


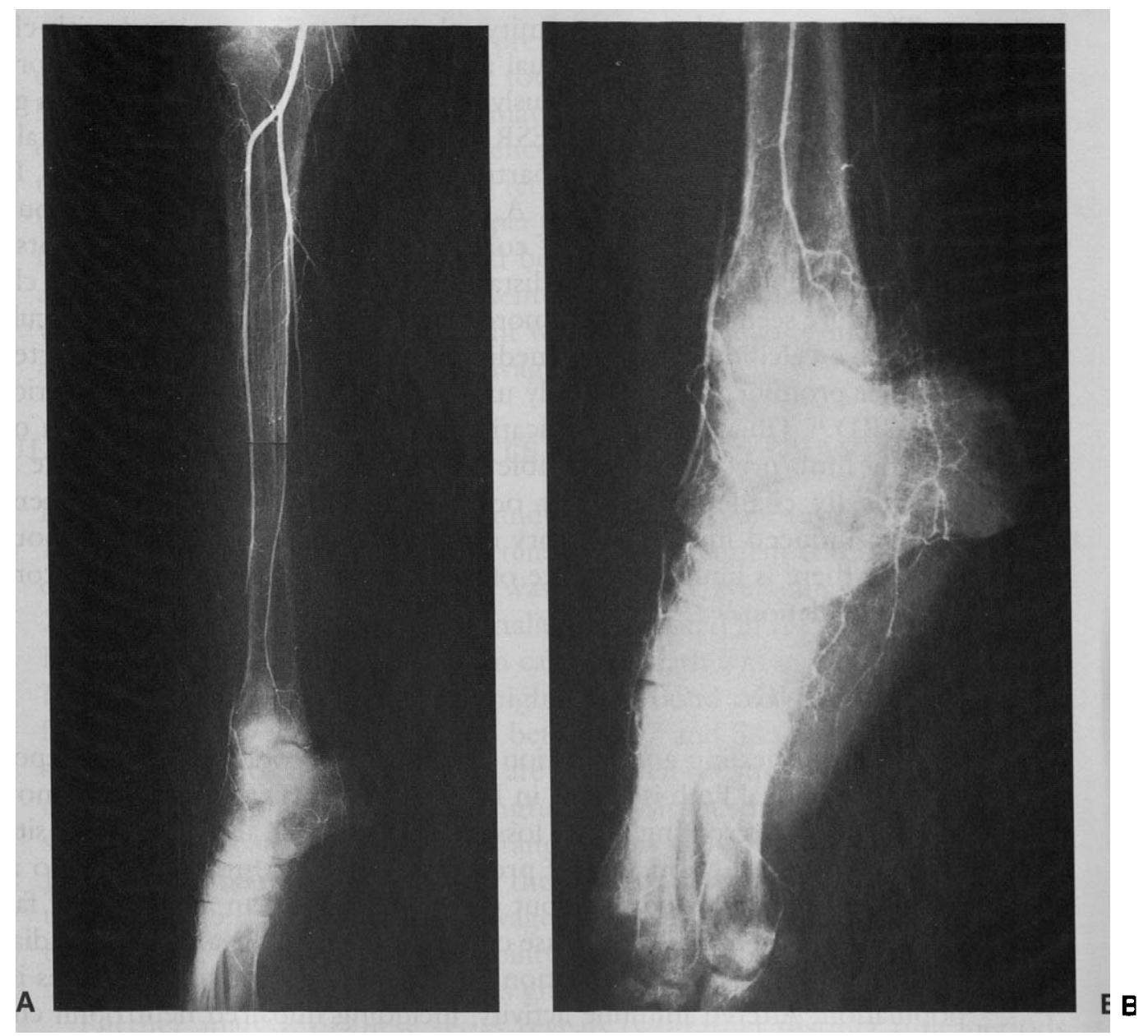

Fig. 1 Angiograms of diabetic ESRD patient with absent toe pressures and forefoot gangrene. (A) The arterial circulation is in continuity to the pedal level with typical "pruning" of the distal tibial vessels. (B) The pedal vasculature is severely affected, and only diseased short segments of major vessels can be identified.

of low-density lipoprotein in uremia. ${ }^{10,11}$ Increased plasma levels of homocysteine has recently been recognized as an independent risk factor for atherosclerosis, and studies have consistently documented elevated concentrations of homocysteine in chronic renal failure, particularly in patients on hemodialysis. ${ }^{12,13}$ Other factors that may play a role in the accelerated atherogenesis of chronic renal failure include secondary hyperparathyroidism and chronic endothelial activation and injury with release of prothrombotic factors. ${ }^{1+15}$ Smoking is especially dangerous in ESRD patients, particularly diabetics in whom smoking more than doubles the rate of myocardial infarction. ${ }^{16}$ 
The pattern of lower extremity atherosclerosis associated with chronic renal failure is somewhat unusual and appears to be less favorable for intervention. Although never rigorously defined in the literature, there is a general recognition that patients with $\mathrm{ESRD}$ have more severe and more distal lower extremity occlusive disease, particularly at the pedal level, than PAOD patients without ESRD (Fig. I A,B). Part of this difference is undoubtedly due to the high incidence of concurrent diabetes mellitus and its welldocumented association with distal disease, although patients with chronic renal failure seem to have even more occlusive disease in the pedal vasculature. Extensive calcification in the media of large, medium, and small arteries is another prominent but not fully understood component of the arteriopathy of ESRD. ${ }^{17}$ Tibial artery calcification makes accurate interpretation of segmental limb pressures impossible in this population and can make IABG technically challenging if not occasionally impossible. The presence of uremia-induced microcirculatory changes has been suggested, although at present there is limited evidence of such derangements only in the coronary microcirculation. ${ }^{11}$

\section{WOUND HEALING}

Poor wound healing and infection are frequent problems in most reports of IABG for critical limb ischemia in ESRD patients. Difficulties occur not only at the site of presenting tissue loss on the foot but also at incision sites for arterial reconstruction. These problems are frequently ascribed to as-yet unproved small artery disease but probably result from a number of factors. Uremia is a well-established cause of impaired wound healing ${ }^{18}$ as are diabetes mellitus, anemia, and malnutrition, which are frequent comorbidities in this population. Altered immune activity, including impaired neutrophil chemotaxis and macrophage function, results from renal failure and undoubtedly plays a role in the reduced ability of these patients to fight infection. ${ }^{19}$ Poor wound healing and inability to contain infection can lead to limb loss despite a functioning bypass, an all-too-frequent complication in many published series. Renal replacement therapy with transplantation avoids many of the problems associated with dialysis (e.g., uremia and anemia), but requires pharmacologic immunosuppression with all its attendant risks for wound healing and infection.

\section{TECHNICAL PROBLEMS ASSOCIATED WITH BYPASS GRAFTING}

There are a number of technical problems associated with lower extremity arterial reconstruction in ESRD patients. The pattern of disease is such that very distal arteries are the best and commonly the only targets for bypass. 
The severe calcification frequently present can challenge even the most proficient surgeon. In situations where tourniquet control is impossible, the utilization of intraluminal occluders may be necessary to avoid clamp injury of calcified distal arteries. The frequency of postoperative incisional complications mandates meticulous surgical technique with avoidance of excessive flap undermining and skin bridges. Upper extremity veins are usually not available for bypass conduits or are avoided because of concerns over future dialysis access. Postoperatively, these patients require a high level of maintenance with careful follow-up and frequent wound debridements and/or local foot amputations for successful limb salvage.

\section{RESULTS OF INFRAINGUINAL ARTERIAL BYPASS GRAFTING}

Published series of ESRD patients undergoing LABG are summarized in Table 1.6,20-32 Diabetes mellitus and hypertension were frequent comorbidities. Perioperative mortality in these reports was substantial, averaging $8.6 \%$ (range 0 to $27 \%$ ), higher than the 2 to $3 \%$ mortality rate quoted in the best series of nonESRD patients. The most common cause of death was myocardial infarction. Perioperative morbidity was also high, and wound complications accounted for the majority, with an incidence between 17 and 54\%. Only 2-year patient survival and limb salvage outcomes are presented because the recognized mortality of ESRD precludes meaningful analysis beyond this point. Patient survival at 2 years averaged $49 \%$ (range 38 to $62 \%$ ), a low survival even for ESRD patients, but a reflection of the severity of associated comorbidities in this population. Two-year limb salvage varied substantially from a low of $50 \%$ to a high of $91 \%$. This wide variability is confusing but important to understand in assessing the role of bypass grafting in this high-risk group.

Further analysis of these series reveals two rather distinct time periods based on outcome (Table 2). The reports from 1988 to 1993 are characterized by an excellent 2 -year limb salvage rate of $79 \%$ (range 71 to $91 \%$ ). In contrast, the 1994 to 1998 series have a much lower average salvage rate of $57 \%$ (range 50 to $62 \%$ ). These data appear to demonstrate worsening IABG results in ESRD patients in the latter part of this decade. Selection and/or reporting bias may partially explain this incongruity, but closer inspection reveals a more likely cause. The patients reported in the later series were older and more frequently diabetic, and by analysis of distal target site appeared to have a greater burden of disease (Table 2). Prior to 1994, bypass was performed to the tibial arteries or lower only $31 \%$ of the time 61 of 199 limbs reported); however, in the later interval (1994 to 1998) a tibial or pedal artery was chosen in $71 \%$ (198 of 278 limbs) of reconstructions. The older age and increasing prevalence of diabetes in patients undergoing IABG in the most recent time period mirrors the changing demographics of ESRD 


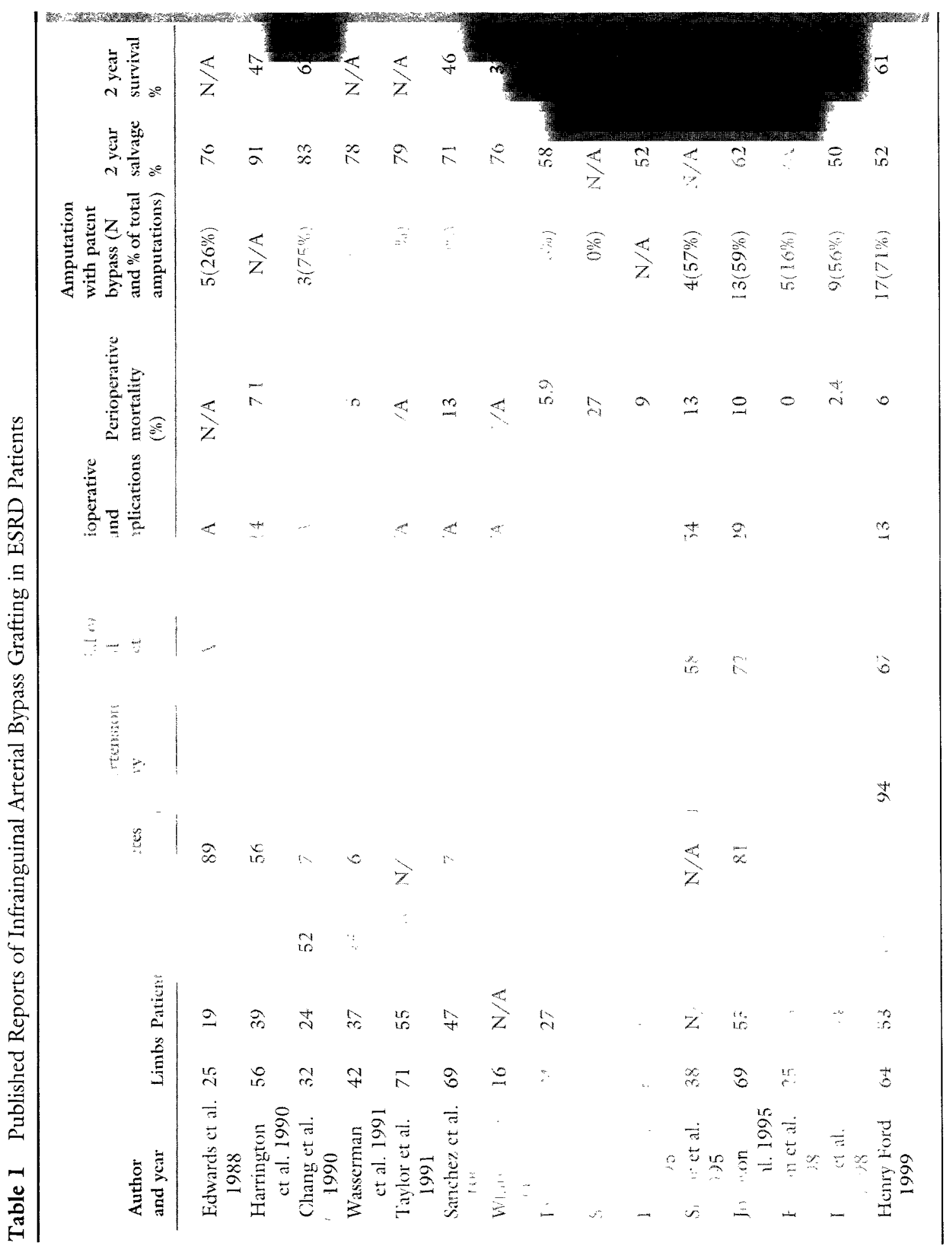


patients in general. ${ }^{5}$ Current results may simply reflect an older, sicker, and more diseased operative cohort.

Limb salvage rates are generally expected to equal or slightly exceed graft patency rates in most IABG reports. However, review of these ESRD series reveals that technical success of the bypass procedure frequently did not equate with limb salvage. In fact, of the 142 amputations performed in these collected reports, 65 (46\%) were performed in the presence of a functioning graft. Thus, roughly $14 \%$ of all infrainguinal reconstructions performed in ESRD limbs will end in amputation despite a patent bypass. Edwards and colleagues were the first to call attention to this phenomenon in $1988 ; 26 \%$ of their ESRD patients required an amputation with a patent graft compared with only $6 \%$ of non-ESRD patients with a functioning bypass. ${ }^{\circ}$ Since this initial report, nearly every large series has documented a similar disappointing outcome in a significant number of patients. ${ }^{20-32}$ Most recently Hakaim and colleagues reviewed their diabetic patients undergoing IABG and found that the amputation rate in the presence of a functioning bypass was significantly higher in ESRD diabetics than in those without renal disease ( $16 \%$ vs $2 \%)^{31}$

Lower extremity amputation in the presence of a patent bypass may reflect the choice of an inappropriate distal target artery that does not provide inline pulsatile flow to the foot. The increasing use of more distal vessels in the 1994 to 1999 era, however, argues against this explanation. Most telling in this regard is the report of Leers et al. in which all patients underwent pedal bypass grafting for tissue loss. ${ }^{32}$ Despite performance of a bypass that guaranteed pulsatile flow to the foot, $22 \%$ of limbs came to amputation with a patent graft. Limb loss in this setting most frequently results from progressive distal necrosis with continued sepsis. ${ }^{6,22,24,26,30,32}$ A less common cause is incisional breakdown and infection with graft exposure requiring graft ligation. ${ }^{7,32}$ Indeed, fear of this devastating complication has led some groups to advocate anatomic tunneling of infrainguinal grafts in ESRD patients in place of the in situ technique. ${ }^{7}$ In either situation of limb loss with a patent graft, problems arise from an inability to heal wounds and clear infection. These

Table 2 Results of Infrainguinal Arterial Bypass Grafting in ESRD Based on Era

\begin{tabular}{lllllllll}
\hline $\begin{array}{l}\text { Inclusive } \\
\text { Years }\end{array}$ & Limbs & $\begin{array}{l}\text { Average } \\
\text { age }\end{array}$ & $\begin{array}{l}\text { Peri- } \\
\text { operative } \\
\text { mortality }(\%)\end{array}$ & $\begin{array}{l}\text { Diabetic } \\
(\%)\end{array}$ & $\begin{array}{l}\text { Hypertensive } \\
(\%)\end{array}$ & $\begin{array}{l}\text { Tibials or 2-year } \\
\text { pedal } \\
\text { target (\%) }\end{array}$ & $\begin{array}{l}\text { 2-year } \\
\text { salvage }\end{array}$ & $\begin{array}{l}\text { survival } \\
(\%)\end{array}$ \\
\hline $1988-1993$ & 311 & 54.5 & 8.5 & 67 & 70 & 31 & 79 & 50 \\
$1994-1998$ & 278 & 61.2 & 8.6 & 85 & 85 & 71 & 57 & 49 \\
\hline
\end{tabular}


data strongly suggest that tissue perfusion and/or wound healing in ESRD patients is impaired well beyond that expected from diabetes alone. ${ }^{31}$

Graft thrombosis rates are not carefully detailed in many of the early ESRD series. Review of later reports where these data are available reveals an average graft thrombosis rate of $14 \%$, most within the first 2 years. ${ }^{6,20-32}$ Although rates are higher than in most series of non-ESRD patients, they are not excessive. The reason for this somewhat higher graft failure rate is unclear. A commonly voiced concern is an increased risk of graft thrombosis during the occasional, unavoidable episodes of hypotension associated with hemodialysis. To date, however, there have been few data in the literature documenting the occurrence of this problem.

\section{PREDICTORS OF OUTCOME}

Most amputations with hemodynamically functioning bypass grafts occur within the first few months following arterial reconstruction. Thus, these high-risk patients are subjected to double jeopardy-the perioperative risks of the bypass procedure and then the amputation-during a relatively short time interval. The benefits of avoiding an unsuccessful attempt at limb salvage are obvious. Several preoperative factors that adversely impact limb salvage after IABG have been identified in the literature, including preoperative infection, ${ }^{28}$ ankle-brachial index $<0.3,{ }^{21}$ poor systemic state,,${ }^{29}$ and history of smoking. ${ }^{32}$ However, none of these factors appears to be a sufficiently strong enough predictor to withhold IABG in otherwise suitable candidates. The degree and location of pedal tissue loss have been particularly closely studied to identify the patient subgroup with a high likelihood of amputation even with technically successful bypass grafting. Edwards et al. identified large ( $>2 \mathrm{~cm}$ in diameter) ischemic ulcerations in diabetic ESRD patients as a bad prognostic sign. ${ }^{6}$ Heel or hindfoot gangrene, defined as full-thickness skin loss $>4 \mathrm{~cm}$ in diameter, was demonstrated by Leers et al. to be a strong negative predictor for both limb salvage and survival. $^{32}$ Even forefoot gangrene, defined as tissue loss beyond the web space of the toes, has been associated with early clinical failure ${ }^{30}$ Although all of these tissue loss predictors seem reasonable, none has been validated in a subsequent large-sçale study.

\section{THE HENRY FORD EXPERIENCE}

To define preoperative factors that might predict the success or failure of IABG in this high-risk group, we recently reviewed our 13-year experience with 53 ESRD patients undergoing 64 infrainguinal bypasses. Patient demographics were similar to those of previously published reports (Table 1). Ninety-one percent of the reconstructions were performed with autogenous 
vein, and the distal target was a tibial or pedal vessel in $67 \%$. All but one patient underwent arterial reconstruction for critical limb ischemia. Perioperative mortality was $6.2 \%$; three of four postoperative myocardial infarctions were fatal. There were seven significant operative wound complications (13\%). The mean duration of follow-up was 22.2 months. At 24 months limb salvage was $52 \%(7.4 \% \mathrm{SE})$, and patient survival was $61 \%(7.3 \% \mathrm{SE})$. There were 24 amputations during follow-up $-7(29 \%)$ were performed for graft failure and 17 (71\%) were performed for uncontrolled infection or progressive tissue loss, despite a patent graft. Fifteen of these 17 amputations occurred within 6 months of IABG.

To evaluate the contribution of pedal level occlusive disease to the phenomenon of amputation with a patent bypass, the arteriograms of 60 of the 64 reconstructions were reviewed, and the pedal runoff was scored using a modified grading scale from 0 to 3 ( 0 reflecting normal pedal runoff and 3 reflecting no named pedal artery patent) as described in the joint vascular surgery societies' revised reporting standards for lower extremity ischemia. ${ }^{33}$ This pedal angiographic resistance score (PARS) was included in an evaluation of factors that might influence limb salvage.

Univariate analysis identified several preoperative factors that adversely impacted limb salvage: age less than 65 years $(p<0.018$, hazard coefficient $4.74)$, preoperative toe pressures less than or equal to $20 \mathrm{mmHg}(p<0.025$, hazard coefficient 2.68$)$, and PARS of 2.5 or greater $(p<0.008$, hazard coefficent 3.14). Smoking, extent of tissue loss, and race were of no significance, although our policy during the latter half of the study period was to not offer patients with extensive tissue loss IABG.

Age less than 65 years as a significant predictor of poor limb salvage is a new finding and may represent more aggressive attempts at limb salvage in younger patients rather than factors present in the limb. The findings that low preoperative toe pressures and a high PARS predict inferior limb salvage in this group are important. By objectively quantitating the extent of occlusive disease in the foot, these measures may predict a patient's ability to heal pedal wounds with a functioning graft. Because the outcome most limiting limb salvage in this group is progressive foot necrosis/infection in the presence of patent bypass, this information could significantly improve IABG results by avoiding revascularization in patients with little chance of success.

Application of these selection criteria (age $<65$ years, low preoperative toe pressures, and high PARS) to the patients in our series would have targeted 12 of 64 limbs for primary amputation rather than bypass. In this group of 12 patients, only 2 had sustained graft patency and limb salvage at 1 year, and 1 of these still has an unhealed heel ulcer at 21 months and is nonambulatory. One patient expired 11 months after reconstruction, 7 underwent amputation with patent bypass grafts within 5 months, and 2 others thrombosed their grafts and underwent amputation within the first vear. 


\section{JUSTIFICATION OF INFRAINGUINAL ARTERIAL BYPASS GRAFTING}

Expected graft patency, limb salvage, and survival following IABG in the ESRD population have been reasonably well established in the recent literature. Available data suggest a perioperative mortality of $9 \%$, a graft thrombosis rate of $14 \%$, and a greater than $14 \%$ amputation rate with a functioning graft. ${ }^{6,20-32}$ Based on these parameters alone, more than one-third of ESRD patients undergoing IABG will have an unacceptable outcome. This sobering figure does not take into account the morbidity and mortality of subsequent amputation and the fact that almost half of these patients will not survive beyond 2 years. In addition, of those patients undergoing "successful" IABG, not all will achieve ambulatory independence- only $31 \%$ in one of the most recently published series. ${ }^{32}$

The results of IABG in ESRD patients are so poor that primary amputation may seem an attractive alternative. Previously, it has been reported that ESRD amputees have similar functional independence, mortality, prosthetic rehabilitation, and cost compared with their non-ESRD counterparts. ${ }^{34}$ However, mortality associated with amputation in this population has been high, ${ }^{8}$ and amputation clearly deprives some patients from the benefits of IABG. The key for improving results is to establish firm guidelines for arterial reconstruction and to move directly to amputation in those patients with little or no chance for limb salvage.

\section{CONCLUSION}

Patients with ESRD probably represent the single most challenging group of patients vascular surgeons are called upon to treat for critical limb ischemia. Results following IABG are far inferior to those in non-ESRD patients. The greatest limitation to acceptable limb salvage in this group is ongoing tissue loss/infection despite a hemodynamically functioning bypass graft. Preoperative factors such as extent of infection, presence of heel ulcers, and poor pedal runoff may help distinguish those patients better served by primary amputation. Further studies to validate the accuracy of current predictors and to develop new ones are desperately needed. Because of the limited number of patients at any one center, the fastest way to accrue such information may be through the creation of a national database. Future research should focus on outcomes other than limb salvage; careful analysis of quality of life parameters, ambulatory status, function, and cost--benefit ratios are essential to develop firm guidelines for IABG in ESRD. Only then can vascular surgeons hope to improve the results of lower extremity revascularization in this very challenging population. 


\section{REFERENCES}

I. Excerpts from the United States Renal Data System 1998 annual data report. Am J Kidney Dis 1998;32:Suppl:S9-S141

2. Abt Associates, Ad Hoc Committee on Nephrology Manpower Needs. Estimated workforce and training requirements for nephrologists through the year 2010 . J Am Soc Nephrol 1997;8:SI-32

3. Luke RG. Chronic renal failure-a vasculopathic state. NEJM 1998;339:841-843

4. United States Renal Data System 1997 Annual Data Report. Bethesda, MD: National Institutes of Health, National Institute of Diabetes and Digestive and Kidney Diseases; $1997 ; 1-33$

5. Herzog CA, Jennie Z, Collins AJ. Poor long-term survival after acute myocardial infarction among patients on long-term dialysis. NEJM 1998;339:799-805

6. Edwards JM, Taylor LM, Porter JM. Limb salvage in end-stage renal disease (ESRD). Arch Surg 1988;123:1164-1168

7. Blakensteijn JD, Gertler JP, Peterson MJ, et al. Avoiding infrainguinal bypass wound complications in patients with chronic renal insufficiency: The role of the anatomic plane. Eur J Vasc Endovasc Surg 1996;11:98-104

8. Dossa CD, Shepard AD, Amos AM, et al. Results of lower extremity amputation in patients with end-stage renal disease. J Vasc Surg 1994;20:14-19

9. Renal Data System. USRD 1997 Annual Data Report. Bethesda, MD: National Institute of Diabetes and Digestive and Kidney Diseases, 1997:D1-D51, 1997. NIH publication no. $97-3176$

10. Maggi E, Bellazzi R, Falaschi F, et al. Enhanced LDL oxidation in uremic patients: An additional mechanism for accelerated atherosclerosis? Kidney Int 1994;45:876-883

11. Becker BN, Himmelfarb J, Henrich WL, et al. Reassessing the cardiac risk profile in chronic hemodialysis patients: A hypothesis on the role of oxidant stress and other nontraditional cardiac risk factors. J Am Soc Neprol 1997;8:475-486

12. Jungers $\mathrm{P}$, Chauveau $\mathrm{P}, \mathrm{Bandin} \mathrm{O}$, ct al. Hyperhomocysteinemia is associated with atherosclerotic occlusive arterial accidents in predialysis chronic renal failure patients. Miner Electrolyte Metab 1997;23:170-173

13. Bachmann J, Tepel $M$, Raidt $H$, et al. Hyperhomocysteinemia and the risk for vascular disease in hemodialysis patients. J Am Soc Nephrol 1995;6:121-125

14. Gris JC, Branger B, Vecina F, et al. Increased cardiovascular risk factors and features of endothelial activation and dysfunction in dialyzed uremic patients. Kidney Int 1994; $46: 807-813$

15. Nishizawa Y, Shoji T, Kawagishi T, Morii H. Atherosclerosis in uremia: Possible roles of hyperparathyroidism and intermediate density lipoprotein accumulation. Kidney Int 1997;62:S90-S92

16. Orth SR, Ritz E, Schrier RW. The renal risks of smoking. Kidney Int 1997;51:1669-1677

17. Rosen H, Friedman SA, Raizener AE, et al. Azotemic arteriopathy. Am Heart J 1972; $84: 250-255$

18. Yue DK, McLennan MM, Mai YW, Spaliviero J, et al. Effects of experimental diabetes, uremia, and malnutrition on wound healing. Diabetes 1987;36:295-299

19. Goldman M, Vanherweghem JL. Bacterial infections in chronic hemodialysis patients: Epidemiologic and pathophysiologic aspects. Adv Nephrol 1990;19:315

20. Chang BB, Paty PSK, Shah DM, et al. Results of infrainguinal bypass for limb salvage in patients with end-stage renal disease. Surgery 1990;108:742-746 
21. Wasserman RJ, Saroyan RM, Rice JC, et al. Infrainguinal revascularization for limb salvage in patients with end-stage renal disease. South Med J 1991;84:190-192

22. Taylor LM, Hamre D, Dalman RL, et al. Limb salvage vs amputation for critical ischemia. Arch Surg 1991;126:1251-1257

23. Harrington $\mathrm{EB}$, Harrington $\mathrm{ME}$, Schanzer $\mathrm{H}$, et al. End-stage renal disease-Is infrainguinal limb revascularization justified? J Vasc Surg 1990;12:691-696

24. Sanchez LA, Goldsmith J, Rivers SP, et al. Limb salvage surgery in end stage renal disease: Is it worthwhile? J Cardiovasc Surg 1992;33:344-348

25. Whittemore $\mathrm{AD}$, Donaldson $\mathrm{MC}$, Mannick JA. Infrainguinal reconstruction for patients with chronic renal insufficiency. J Vasc Surg 1993;17:32-41

26. Lumsden $A B$, Besman $A$, Jaffe $M$, et al. Infrainguinal revascularization in end-stage renal disease. Ann Vasc Surg 1994;8:107-112

27. Sakurai $T$, Kobayashi $M$, Harasawa $H$, et al. Infrainguinal arterial reconstruction in endstage renal disease. Cardiovasc Surg 1995;3:46-49

28. Baele HR, Piotrowski JJ, Yuhas J, Anderson C, Alexander JJ. Infrainguinal bypass in patients with end-stage renal disease. Surgery 1995;117:319-324

29. Simsir SA, Cabellon A, Kohlman-Trigoboff D, et al. Factors influencing limb salvage and survival after amputation and revascularization in patients with end-stage renal disease. Am J Surg 1995;170:113-117

30. Johnson BL, Glickman MH, Bandyk DF, Esses GE. Failure of foot salvage in patients with end-stage renal disease after surgical reconstruction. J Vasc Surg 1995;22:280-286

31. Hakaim AG, Gordon JK, Scott TE. Early outcome of in situ femorotibial reconstruction among patients with diabetes alone versus diabetes and end-stage renal failure: Analysis of 83 limbs. J Vasc Surg 1998;27:1049-1055

32. Leers SA, Reifsnyder T, Delmonte R, et al. Realistic expectations for pedal bypass grafts in patients with end-stage renal disease. J Vasc Surg 1998;28:976-983

33. Rutherford RB, Baker D, Ernst C, et al. Recommended standards for reports dealing with lower extremity ischemia: Revised version. J Vasc Surg 1997;26:517-538

34. Czyrny JJ, Merrill A. Rehabilitation of amputees with end-stage renal disease-Functional outcome and cost. Am J Phys Med Rehabil 1994;73:353-357 